\title{
Relación entre ideación suicida y estilos parentales en un grupo de adolescentes chilenos
}

\author{
RAMÓN FLORENZANO U. ${ }^{1,2,3}$, MACARENA VALDÉS C. ${ }^{2, a}$, \\ EUGENIO CÁCERES C. ${ }^{3, \mathrm{~b}}$, SYLVIA SANTANDER R. ${ }^{4}$, \\ CAROLINA ASPILLAGA H. ${ }^{1, \mathrm{c}}$, CLAUDIA MUSALEM A. ${ }^{1, \mathrm{~d}}$
}

\section{Relation between suicidal ideation and parenting styles among a group of Chilean adolescents}

Background: In Chile, there has been an increase in suicide rates from 1.1 to 2.6 per 100,000 among adolescents aged 10 to 14 years and from 4.4 to 8.9 per 100,000 among those aged 15 to 19 years Aim: To identify protective factors for suicidal ideation according to parenting styles, as described by Barber et al. Material and Methods: The relation between suicidal ideation and parenting styles was assessed in a random sample of 2,346 Chilean school attending adolescents aged 13 to 20 years old (59\% women) from three cities: Antofagasta (Northern Chile, II Region), Santiago (Central, Metropolitan Region) and Concepción (Southern, VIII Region). Participants were tested with the Chilean adaptation of the Cross National Adolescents Program (CNAP) Plus questionnaire developed by Barber et al. The relation between suicidal ideation and parenting styles was assessed using regression analyses. Results: Correlations between suicidal ideation and parenting styles were mostly significant, yet weak. High odds ratios were observed among parents who had a strong psychological control, inconsistent control, lack of expression of affection and covered marital hostility. Conclusions: High adolescent self-esteem, a good relationship with parents, psychological parental autonomy, expression of physical affection, social support and paternal monitoring were protective factors against suicidal ideation.

(Rev Med Chile 2011; 139: 1529-1533).

Key words: Adolescent; Parents; Risk factors; Suicide.

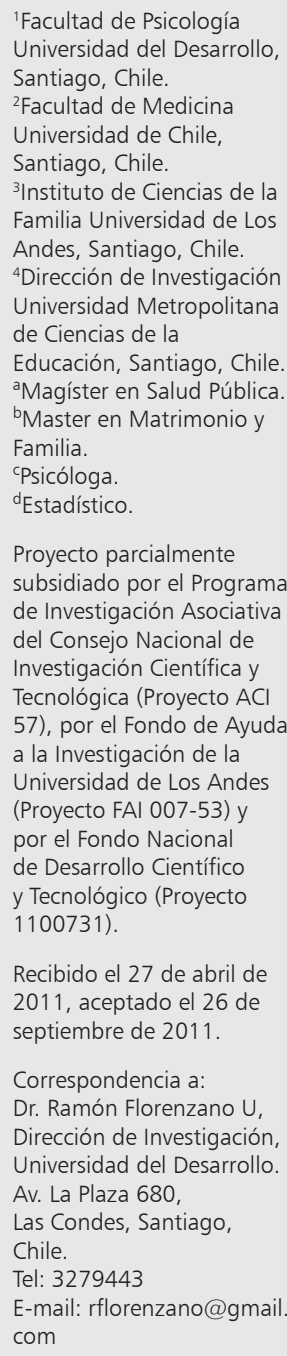

I os problemas de salud mental de los adolescentes, y las conductas que de éstos se desprenden, están asociados a factores genéticos y ambientales o del contexto en que viven. Los estilos parentales han sido estudiados junto a la relación con los pares, los vecinos y el entorno escolar; para ello, Barber et al han propuesto un modelo interaccional ${ }^{1}$ que ha sido validado transculturalmente usando la batería de escalas Cross National Adolescents Program (CNAP). En ella se describen las diversas dimensiones de estilos parentales y sus efectos entre niños y adolescentes. Según Barber et al, la parentalidad está compuesta por las dimensiones de aceptación, apoyo y control, esta última en la doble referencia de psicológico y conductual. El primero se relaciona con el desarrollo psicológico y emocional del niño adolescente, como invalidación de sentimientos, restricción de expresiones verbales, retiro de amor e inducción de culpabilidad entre otras. Esta dimensión se relaciona con depresión y conductas antisociales en los adolescentes. El control conductual indica el grado en que los padres establecen reglas y regulaciones que las hacen cumplir, poniendo límites a las actividades del adolescente. Esta dimensión se relaciona con menores comportamientos antisociales en los adolescentes. Las conductas parentales de acepta- 
ción y apoyo se correlacionan positivamente con la competencia interpersonal de los adolescentes y sus conductas prosociales, mientras que el control psicológico autoritario se correlacionaría positivamente con una menor salud mental del adolescente, y en especial con síntomas depresivos. El control conductual parental se correlacionaría, a su vez, con menos conducta antisocial entre los adolescentes. Este tipo de control parental facilita la autorregulación y fomenta conductas socialmente aceptadas en los adolescentes. Aquéllos sin suficiente supervisión parental están más expuestos a ser influenciados por sus pares, en especial por aquellos que ejercen un liderazgo negativo, promoviendo conductas desviadas y peligrosas. Este modelo ha sido correlacionado empíricamente en un estudio transcultural en 16 países $^{2}$ y ha sido replicado en Chile, en un estudio desarrollado por un grupo de investigadores de cuatro universidades chilenas, quienes trabajaron en conjunto con el Profesor Barber entre 2006 y 2008.

Uno de los mayores problemas que se encuentra en los adolescentes actualmente tiene relación con la ideación, plan e intento suicida, conducta que contribuye en forma importante a su morta$\operatorname{lidad}^{3}$. La evolución de las tasas de suicidio entre los jóvenes en las últimas décadas en Chile ha aumentado: María Inés Romero señala (datos no publicados que entre los 10 y 14 años se registró un alza de 1,1 a 2,6 por 100 mil habitantes, mientras que en jóvenes de 15 a 19 años la tasa aumentó de 4,4 a 8,9 por 100 mil. En cifras absolutas, los suicidios adolescentes registrados desde 1983 hasta 2003 fueron de 5.924 jóvenes. La Encuesta Mundial de Salud Escolar reveló que 20\% de los jóvenes chilenos ha considerado seriamente suicidarse, mientras que entre 15,3 y $16,5 \%$ de ellos ha hecho planes sobre cómo hacerlo ${ }^{4}$

El objetivo de esta publicación es establecer factores protectores de la ideación suicida dentro de las redes familiares, para identificar de manera concreta aquellos estilos parentales que se relacionan con la ideación suicida y difundirlos con afán preventivo.

\section{Material y Método}

Se realizó un muestreo aleatorio tri-etápico en 27 escuelas escogidas de un listado nacional confeccionado por el Ministerio de Educación de Chile ${ }^{5}$, seleccionando en primer lugar comunas, luego, escuelas y finalmente, clases. El sistema educacional chileno divide las escuelas en tres tipos, según su régimen administrativo, municipales, particulares subvencionadas y particulares pagadas. En la Tabla 1 se presenta la distribución de los estudiantes encuestados por Región, tipo de colegio y nivel socioeconómico (NSE). De las 27 escuelas muestreadas, nueve eran municipales, ocho subvencionadas y diez privadas.

La encuesta aplicada fue anónima y voluntaria. El cuestionario fue aplicado por personas entrenadas a 2.346 alumnos durante 2007 a 2009. El instrumento de evaluación utilizado fue una adaptación en castellano (CNAP Plus) de la escala CNAP desarrollada en un estudio transcultural por Barber et $\mathrm{al}^{2}$ entre 1999 y 2004. La adaptación la realizaron los autores en tres reuniones de trabajo conjuntas con el profesor Barber (Bellagio, Italia-2006; Santiago y Concepción, Chile-2007). Las edades de los estudiantes muestreados se incluían en el rango 13-20. El promedio de edad fue de 15,6 $\pm 1,25$ años. El 55,8\% de los encuestados eran mujeres.

Los Comités de Ética de la Universidad del Desarrollo y de la Clínica Alemana revisaron la metodología, los consentimientos informados y la confidencialidad de los datos y aprobaron el estudio. El protocolo de investigación y el cuestionario CNAP Plus fueron presentados a la Junta Institucional de Revisión de la Universidad de Los Andes, quienes aceptaron dicho cuestionario de consentimiento informado.

La ideación suicida se midió a través de la declaración: 'He pensado en formas de matarme', las respuestas posibles eran: 'no es cierto/no se aplica', 'a veces cierto/algo cierto' y 'muy cierto/a menudo cierto'. Las variables referentes a estilos parentales

Tabla 1. Resultados tablas de contingencia entre ideación suicida y variables demográficas, estudiantes de 3 ciudades, Chile, 2007-2009

\begin{tabular}{|lccc|}
\hline Variables & $\mathbf{n}^{\mathbf{a}}$ & $\begin{array}{c}\text { Coeficiente de } \\
\text { Contingencia }\end{array}$ & $\mathbf{P}^{\mathbf{b}}$ \\
\hline Tipo de colegio & 2.316 & 0,079 & $0,006^{*}$ \\
\hline Género & 2.295 & 0,078 & $0,001^{*}$ \\
\hline Región de Chile & 2.316 & 0,093 & $0,000^{*}$ \\
\hline NSE & 2.316 & 0,113 & $0,000^{*}$ \\
\hline Edad & $2 . .236$ & 0,100 & 0,065 \\
\hline
\end{tabular}

aCantidad de casos válidos, 'Valor de significación bilateral, *Valor P significativo. 
Ideación suicida y estilos parentales en adolescentes - R. Florenzano et al

corresponden a todas aquellas propuestas por el modelo de Barber et $\mathrm{al}^{1}$ sobre el cual se basó este estudio.

Para todos los análisis estadísticos los valores perdidos se excluyeron de a parejas. De la base completa hubo 2.287 alumnos que respondieron las preguntas sobre ideación suicida y depresión de Achenbach. Estos se dividieron en dos subgrupos: aquellos estudiantes con síntomas depresivos $(\mathrm{n}=$ $352)$ y aquéllos sin éstos $(n=1.935)$. Se prosiguió a calcular las correlaciones entre ideación suicida y estilos parentales para todos los estudiantes pertenecientes a cada partición. Como coeficiente de correlación se utilizó Rho de Spearman con significación bilateral.

Posteriormente, se realizó una regresión logística donde la variable dependiente fue: ' $\mathrm{He}$ pensado en formas de matarme'. La variable sobre ideación suicida se dicotomizó en una escala 'nunca cierto/no se aplica' y 'algo/a veces/muy/a menudo cierto'. Las razones de posibilidades u odds ratio $(\exp (\beta))$ se trabajaron con un intervalo de confianza de $95 \%$.

\section{Resultados}

Inicialmente, se revisaron las respuestas a la pregunta 'He pensado en formas de matarme': el 6,4\% respondió 'muy cierto/a menudo cierto', el $22,2 \%$ respondió 'algo cierto/a veces cierto' y el $71,4 \%$ restante respondió 'no es cierto/no se aplica'.

Luego, mediante tablas de contingencia, se revisó la asociación existente entre la variable de ideación suicida y género, región, NSE, tipo de colegio y edad. Todas arrojaron una asociación significativa (mayor a 0,05 ) respecto a la ideación suicida excepto por la variable edad, como se puede apreciar en la Tabla 1.

Para clasificar a los alumnos según presencia de síntomas depresivos se utilizó la variable depresión de Achenbach. En la Tabla 2, se muestran las correlaciones entre ideación suicida y estilos parentales que resultaron negativas dentro de los alumnos con síntomas depresivos. Es decir, se muestran las dimensiones que incitarían una menor ideación suicida para los alumnos que presentan síntomas depresivos. En la Tabla 3 se entregan las correlaciones entre ideación suicida y estilos parentales para los alumnos sin síntomas depresivos. Aunque son débiles, las correlaciones indican que existiría una relación inversa entre ideación suicida y las dimensiones de estilos parentales. A medida que mejoran la ideación suicida disminuye, es decir, a mayor aceptación, apoyo y control es menor la ideación suicida.

Para los alumnos con síntomas depresivos

Tabla 2. Correlaciones negativas entre ideación suicida y variables estilos parentales de alumnos con síntomas depresivos, estudiantes de 3 ciudades, Chile, 2007-2009

\begin{tabular}{|lcc|}
\hline Variables & Rho $^{\mathbf{a}}$ & \multicolumn{1}{c|}{$\mathbf{P}^{\mathbf{b}}$} \\
\hline Autonomía psicológica parental & $-0,233 \leq 0,01$ \\
Calidad relación con el padre & $-0,198 \leq 0,01$ \\
Calidad relación con la madre & $-0,186 \leq 0,01$ \\
\hline Amabilidad parental & $-0,148 \leq 0,05$ \\
\hline Participación en decisiones familiares & $-0,137 \leq 0,05$ \\
\hline Aceptación parental & $-0,123 \leq 0,05$ \\
\hline Expresión de afecto parental físico & $-0,123 \leq 0,05$ \\
\hline Autoestima & $-0,118 \leq 0,05$ \\
\hline Monitoreo paterno & $-0,116 \leq 0,05$ \\
\hline Toma decisiones con la madre & $-0,112 \leq 0,05$ \\
\hline
\end{tabular}

${ }^{a}$ Rho de Spearman, ${ }^{b}$ Valor de significación bilateral.

Tabla 3. Correlaciones negativas entre ideación suicida y variables estilos parentales de alumnos sin síntomas depresivos, estudiantes de 3 ciudades, Chile, 2007-2009

\begin{tabular}{lcc|}
\hline Variables & Rho $^{\mathbf{a}}$ & \multicolumn{1}{c|}{$\mathbf{P b}^{\mathbf{b}}$} \\
\hline Autoestima & $-0,161$ & $\leq 0,01$ \\
\hline Aceptación parental & $-0,093 \leq 0,01$ \\
\hline Autonomía psicológica parental & $-0,092 \leq 0,01$ \\
\hline Calidad relación con la madre & $-0,084 \leq 0,01$ \\
\hline Regulación escolar & $-0,082 \leq 0,01$ \\
Calidad relación con el padre & $-0,076 \leq 0,01$ \\
\hline Deseo de participar en decisiones & & \\
familiares & $-0,074 \leq 0,01$ \\
\hline Amabilidad parental & $-0,074 \leq 0,01$ \\
\hline Familia servicial & $-0,070 \leq 0,01$ \\
\hline Control conductual parental & $-0,068 \leq 0,01$ \\
\hline Expresión de afecto parental físico & $-0,065 \leq 0,01$ \\
\hline Horas tareas & $-0,063 \leq 0,01$ \\
\hline Iniciativa social & $-0,055 \leq 0,05$ \\
\hline Monitoreo materno & $-0,052 \leq 0,05$ \\
\hline Monitoreo paterno & $-0,052 \leq 0,05$ \\
\hline Participación en decisiones familiares & $-0,047 \leq 0,05$ \\
\hline
\end{tabular}

aRho de Spearman, bValor de significación bilateral. 
Tabla 4. Análisis de Regresión Logística de Variables Modelo Barber prediciendo Ideación Suicida, estudiantes de tres ciudades, Chile, 2007-2009

\begin{tabular}{|c|c|c|c|c|}
\hline \multirow{2}{*}{$\begin{array}{l}\text { Variables } \\
\text { Control Psicológico Parental }\end{array}$} & \multirow{2}{*}{$\begin{array}{l}\text { Nivel Variable } \\
\text { Alto Control } \\
\text { Bajo Control }\end{array}$} & \multicolumn{2}{|c|}{ RP $^{a}\left(I^{b}\right.$ 95\%) } & \multirow{2}{*}{$\begin{array}{c}\mathbf{p}^{\mathbf{c}} \\
0,00\end{array}$} \\
\hline & & $\begin{array}{c}1,78 \\
1\end{array}$ & $(1,48-2,15)$ & \\
\hline Control Parental Inconsistente & $\begin{array}{l}\text { Alto Control } \\
\text { Bajo Control }\end{array}$ & $\begin{array}{c}1,60 \\
1\end{array}$ & $(1,33-1,93)$ & 0,00 \\
\hline Autonomía Psicológica Parental & $\begin{array}{l}\text { Baja Autonomía } \\
\text { Alta Autonomía }\end{array}$ & $\begin{array}{c}2,00 \\
1\end{array}$ & $(1,67-2,40)$ & 0,00 \\
\hline Aceptación Parental & $\begin{array}{l}\text { Baja Aceptación } \\
\text { Alta Aceptación }\end{array}$ & $\begin{array}{c}2,17 \\
1\end{array}$ & $(1,80-2,61)$ & 0,00 \\
\hline Expresión de Afecto Parental Físico & $\begin{array}{l}\text { Poco Afecto } \\
\text { Mucho Afecto }\end{array}$ & $\begin{array}{c}1,77 \\
1\end{array}$ & $(1,47-2,14)$ & 0,00 \\
\hline Amabilidad Parental & $\begin{array}{l}\text { Baja Amabilidad } \\
\text { Alta Amabilidad }\end{array}$ & $\begin{array}{c}1,97 \\
1\end{array}$ & $(1,64-2,36)$ & 0,00 \\
\hline Crueldad Parental & $\begin{array}{l}\text { Alta Crueldad } \\
\text { Baja Crueldad }\end{array}$ & $\begin{array}{c}1,98 \\
1\end{array}$ & $(1,62-2,42)$ & 0,00 \\
\hline Monitoreo Paterno & $\begin{array}{l}\text { Bajo Monitoreo } \\
\text { Alto Monitoreo }\end{array}$ & $\begin{array}{c}1,59 \\
1\end{array}$ & $(1,32-1,91)$ & 0,00 \\
\hline Calidad Relación con la Madre & $\begin{array}{l}\text { Pobre Relación } \\
\text { Buena Relación }\end{array}$ & $\begin{array}{c}2,22 \\
1\end{array}$ & $(1,83-2,71)$ & 0,00 \\
\hline Calidad Relación con el Padre & $\begin{array}{l}\text { Pobre Relación } \\
\text { Buena Relación }\end{array}$ & $\begin{array}{c}2,11 \\
1\end{array}$ & $(1,8-2,5)$ & 0,00 \\
\hline Participación Decisiones Familiares & $\begin{array}{l}\text { Baja Participación } \\
\text { Alta Participación }\end{array}$ & $\begin{array}{c}1,61 \\
1\end{array}$ & $(1,34-1,93)$ & 0,00 \\
\hline Hostilidad Marital Encubierta & $\begin{array}{l}\text { Alta Hostilidad } \\
\text { Baja Hostilidad }\end{array}$ & $\begin{array}{c}2,51 \\
1\end{array}$ & $(2,07-3,06)$ & 0,00 \\
\hline Comportamiento Antisocial & $\begin{array}{l}\text { Alto Comportamiento } \\
\text { Bajo Comportamiento }\end{array}$ & $\begin{array}{c}2,46 \\
1\end{array}$ & $(2,05-2,96)$ & 0,00 \\
\hline Autoestima & $\begin{array}{l}\text { Baja Autoestima } \\
\text { Alta Autoestima }\end{array}$ & $\begin{array}{c}2,30 \\
1\end{array}$ & $(1,89-2,79)$ & 0,00 \\
\hline
\end{tabular}

aRP: Razón de Posibilidades. ${ }^{b} \mathrm{IC}$ : Intervalo de Confianza. 'Valor p de Significación > 0,05.

el tomar decisiones con la madre es un factor protector, mientras que para los alumnos sin síntomas depresivos no lo es. Para los alumnos sin síntomas depresivos la regulación escolar, el deseo de participar en decisiones familiares, las horas de tareas, la iniciativa social, el monitoreo materno, el control conductual parental y el pertenecer a una familia servicial actúan como factores protectores, mientras que para los alumnos con síntomas depresivos estas variables no aparecen como factores protectores. Estas diferencias se pueden observar comparando las Tablas 2 y 3.

El análisis de regresión logística mostró que aquellos adolescentes con padres que presentan un alto nivel de hostilidad marital encubierta tienen una razón de posibilidades más alto de ideación suicida $(2,51$ veces más que los adolescentes con padres sin hostilidad marital encubierta). Los alumnos con alto comportamiento antisocial tienen 2,46 veces más posibilidades de presentar ideación suicida que aquellos adolescentes que tienen un adecuado comportamiento antisocial. Los adolescentes con baja autoestima presentan 2,3 veces más prosibilidades de ideación suicida que aquellos alumnos con alta autoestima. Estos resultados aparecen en la Tabla 4.

\section{Discusión}

$\mathrm{Al}$ no existir una asociación estadísticamente significativa entre ideación suicida y edad, puede estimarse que la primera no se vincula necesariamente a las diversas etapas de crecimiento cronológico del adolescente. En otra publicación, hemos descrito la correlación entre estilos parentales según NSE y ubicación geográfica, pudiendo ob- 
servarse que la ideación suicida es mayor en establecimientos municipales ( $8,2 \%)$, en comparación con los privados (5,5\%). Asimismo, las conductas hetero-agresivas (robo, vandalismo o riñas) son más frecuentes en establecimientos municipales que en privados ${ }^{6}$. No hubo correlación estadística entre ciudad y suicidalidad.

Se analizaron las correlaciones existentes entre ideación suicida y estilos parentales, tanto para adolescentes con y sin síntomas depresivos: dentro de las correlaciones significativas, para los alumnos sin síntomas depresivos aparecieron más factores protectores de ideación suicida relacionados con los estilos parentales que para los alumnos con síntomas. Se registraron nueve factores protectores en común para ambos grupos: autoestima, aceptación parental, autonomía psicológica parental, calidad en la relación con la madre, calidad en la relación con el padre, amabilidad parental, expresión de afectos físicos, monitoreo paterno y participación en decisiones familiares. Estos resultados revelan que la ideación suicida tiene una mayor correlación con los estilos parentales que con la edad o etapas de la adolescencia. Los adolescentes cuyos padres ejercen autonomía psicológica, aceptación parental, expresión de afectos físicos, amabilidad parental y monitoreo paterno poseen menor ideación suicida que los adolescentes cuyos padres carecen de estas conductas.

En cuanto a la relación de los padres con sus hijos adolescentes, un factor protector para la ideación suicida es que los adolescentes tengan una buena relación con ambos padres. También actúa como factor protector la participación de los hijos en las decisiones familiares.

Para aquellos jóvenes con alto comportamiento antisocial y baja autoestima, las posibilidades de desarrollar ideación suicida son mayores que para aquellos jóvenes con alta autoestima y bajo comportamiento antisocial. Estos resultados muestran que los estilos parentales influyen de manera sustancial en la ideación suicida de los jóvenes. Para los adolescentes, la relación entre sus padres, los estilos de crianza parental que imponen y la relación que desarrollan los padres con ellos logra disminuir o aumentar la presencia de ideación suicida. Los hallazgos de este estudio subrayan la necesidad de crear conciencia entre los padres respecto a su influencia en la salud de los hijos, y de entregarles herramientas concretas de conductas y estilos de crianza protectores. En este contextos es relevante la participación de entidades académicas y otras en la generación de adecuadas bases de información y de programas de formación profesional. Finalmente, este estudio apunta a la influencia central de la familia como factor de riesgo en el aumento de la ideación suicida adolescente. Tal como es parte del problema, la familia debiera considerarse como un elemento central en su solución.

Agradecimientos: Se agradece el apoyo económico del Programa de Investigación Asociativa del Consejo Nacional de Investigación Científica y Tecnológica (Proyecto ACI 57) y del Fondo de Ayuda a la Investigación de la Universidad de Los Andes, quienes otorgaron subsidios a la investigación que permitieron realizar este estudio.

\section{Referencias}

1. Barber BK (Ed.). Intrusive parenting: How psychological control affects children and adolescents. Washington, DC: American Psychological Association Press. 2002.

2. Barber BK, Stolz HE, Olsen JA. Parental support, Psychological control and Behavioral control: Assessing relevance across time, methods and culture, Monogr Soc Res Child Dev 2005; 70 N. 4.

3. Florenzano R, Valdés M. Conductas de riesgo adolescentes y factores protectores. En: Florenzano, Valdés eds. El Adolescente y sus Conductas de Riesgo. Ediciones Universidad Católica de Chile. Tercera Edición 2005.

4. Encuesta Mundial de Salud Escolar Chile 2004 y 2005; 18. Disponible en: http://epi.minsal.cl/epi/html/invest/ EMSE/INFORME\%20EMSE\%202004-2005\%20final. pdf [Consultado el 19 de agosto de 2010].

5. Ministerio de Educación, Chile. SIMCE. Metodología de Construcción de Grupos Socio-Económicos en $2^{\circ}$ Medio 2008. Santiago, 2009.

6. Florenzano R, Valdés M, Cáceres E, Cassasus M, Sandoval A, Santander S, et al. Comparación de la Relación Parental entre Mayores y Menores de 15 años. Rev Chil Pediatr 2009; 80 (6): 519-26.

7. Barber BK, Olsen JA. Socialization in context: Connection, regulation, and autonomy in the family, school, and neighborhood, and with peers. J Adolesc Res 1997; 12 (2): 287-315.

8. Florenzano R, Cáceres E, Valdés M, Calderón S, Santander S, Cassasus M, et al. Comparación de frecuencia de conductas de riesgo, problemas juveniles y estilos de crianza, en estudiantes adolescentes de tres ciudades chilenas. Cuad Med Soc (Chile) 2010; 50 (2): 115-23. 